\title{
Determination of a Control Parameter for the Difference Schrödinger Equation
}

\author{
Allaberen Ashyralyev ${ }^{1,2}$ and Mesut Urun ${ }^{1,3}$ \\ ${ }^{1}$ Department of Mathematics, Fatih University, Buyukcekmece, 34500 Istanbul, Turkey \\ ${ }^{2}$ ITTU, 32 Gerogly Street, 74400 Ashgabat, Turkmenistan \\ ${ }^{3}$ Department of Mathematics, Murat Education Institution, 34353 Istanbul, Turkey
}

Correspondence should be addressed to Mesut Urun; mesuturun@gmail.com

Received 28 July 2013; Accepted 18 September 2013

Academic Editor: Abdullah Said Erdogan

Copyright (C) 2013 A. Ashyralyev and M. Urun. This is an open access article distributed under the Creative Commons Attribution License, which permits unrestricted use, distribution, and reproduction in any medium, provided the original work is properly cited.

The first order of accuracy difference scheme for the numerical solution of the boundary value problem for the differential equation with parameter $p, i(d u(t) / d t)+A u(t)+i u(t)=f(t)+p, 0<t<T, u(0)=\varphi, u(T)=\psi$, in a Hilbert space $H$ with self-adjoint positive definite operator $A$ is constructed. The well-posedness of this difference scheme is established. The stability inequalities for the solution of difference schemes for three different types of control parameter problems for the Schrödinger equation are obtained.

\section{Introduction: Difference Scheme}

The theory and applications of well-posedness of inverse problems for partial differential equations have been studied extensively in a large cycle of papers (see, e.g., $[1-24]$ and the references therein).

Our goal in this paper is to investigate Schrödinger equations with parameter. In the paper [25], the boundary value problem for the differential equation with parameter $p$

$$
\begin{gathered}
i \frac{d u(t)}{d t}+A u(t)+i u(t)=f(t)+p, \quad 0<t<T, \\
u(0)=\varphi, \quad u(T)=\psi
\end{gathered}
$$

in a Hilbert space $H$ with self-adjoint positive definite operator $A$ was studied. The well-posedness of this problem was established. The stability inequalities for the solution of three determinations of control parameter problems for the Schrödinger equation were obtained. In the present paper, the first order of accuracy Rothe difference scheme

$$
\begin{gathered}
i \tau^{-1}\left(u_{k}-u_{k-1}\right)+A u_{k}+i u_{k}=\varphi_{k}+p, \quad \varphi_{k}=f\left(t_{k}\right), \\
t_{k}=k \tau, \quad 1 \leq k \leq N, \quad N \tau=T, \\
u_{0}=\varphi, \quad u_{N}=\psi
\end{gathered}
$$

for the approximate solution of the boundary value problem (1) for the differential equation with parameter $p$ is presented. It is easy to see that

$$
\begin{aligned}
& u_{k}=v_{k}+(A+i I)^{-1} p, \\
& p=(A+i I)\left(\psi-v_{N}\right),
\end{aligned}
$$

where $\left\{v_{k}\right\}_{k=0}^{N}$ is the solution of the following single-step difference scheme:

$$
\begin{gathered}
i \tau^{-1}\left(v_{k}-v_{k-1}\right)+A v_{k}+i v_{k}=\varphi_{k}, \quad \varphi_{k}=f\left(t_{k}\right), \\
t_{k}=k \tau, \quad 1 \leq k \leq N, \quad N \tau=T, \\
v_{0}-v_{N}=\varphi-\psi .
\end{gathered}
$$

The theorem on well-posedness of difference problem (2) is proved. In practice, the stability inequalities for the solution of difference schemes for the approximate solution of three different types of control parameter problems are obtained.

The paper is organized as follows. Section 1 is the introduction. In Section 2, the main theorem on stability of difference problem (2) is established. In Section 3, theorems on the stability inequalities for the solution of difference schemes for the approximate solution of three different types of control 
parameter problems are obtained. In Section 4, numerical results are given. Finally, Section 5 is the conclusion.

\section{The Main Theorem on Stability}

In this section, we will study the stability of difference scheme (2).

Let $[0, T]_{\tau}=\left\{t_{k}=k \tau, k=1, \ldots, N, N \tau=T\right\}$ be the uniform grid space with step size $\tau>0$, where $N$ is a fixed positive integer. Throughout the present paper, $\mathscr{F}\left([0, T]_{\tau}, H\right)$ denotes the linear space of grid functions $\varphi^{\tau}=\left\{\varphi_{k}\right\}_{1}^{N}$ with values in the Hilbert space $H$. Let $\mathscr{C}_{\tau}(H)=\mathscr{C}\left([0, T]_{\tau}, H\right)$ be the Banach space of bounded grid functions with the norm

$$
\left\|\varphi^{\tau}\right\|_{\mathscr{C}_{\tau}(H)}=\max _{1 \leq k \leq N}\left\|\varphi_{k}\right\|_{H^{*}}
$$

Let us start with a lemma we need below. We denote that $R=$ $((1+\tau) I-i \tau A)^{-1}$ is the step operator of problem (2).

Lemma 1. Assume that $A$ is a positive definite self-adjoint operator. The operator $I-R^{N}$ has an inverse $T_{\tau}=\left(I-R^{N}\right)^{-1}$ and the following estimate is satisfied:

$$
\left\|T_{\tau}\right\|_{H \rightarrow H} \leq M(\delta) .
$$

Proof. The proof of estimate (6) is based on the triangle inequality and the estimate

$$
\begin{aligned}
\left\|\left(I-R^{N}\right)^{-1}\right\|_{H \rightarrow H} & \leq \sup _{\delta \leq \mu} \frac{1}{1-\left|(1+\tau(1-i \mu))^{-N}\right|} \\
& \leq \frac{1}{1-\left((1+\tau)^{2}+(\tau \delta)^{2}\right)^{-N / 2}} \leq \mu(\delta) .
\end{aligned}
$$

Now, let us obtain the formula for the solution of problem (2). It is clear that the first order of accuracy difference scheme

$$
\begin{gathered}
i \tau^{-1}\left(u_{k}-u_{k-1}\right)+A u_{k}+i u_{k}=p+\varphi_{k}, \quad \varphi_{k}=f\left(t_{k}\right), \\
t_{k}=k \tau, \quad 1 \leq k \leq N, \quad N \tau=T, \quad u_{0}=\varphi
\end{gathered}
$$

has a solution and the following formula

$$
u_{k}=R^{k} \varphi-i \sum_{j=1}^{k} R^{k-j+1}\left(p+\varphi_{j}\right) \tau, \quad 1 \leq k \leq N
$$

is satisfied. Applying formula (9) and the boundary condition

$$
u_{N}=\psi,
$$

we can write

$$
\psi=R^{N} \varphi-i \sum_{j=1}^{N} R^{N-j+1} \varphi_{j} \tau-i \sum_{j=1}^{N} R^{N-j+1} \tau p .
$$

Since

$$
\begin{aligned}
-i \sum_{j=1}^{N} R^{N-j+1} \tau & =-i(I-i A)^{-1}(I-R) \sum_{j=1}^{N} R^{N-j} \\
& =-i(I-i A)^{-1}\left(I-R^{N}\right),
\end{aligned}
$$

we have that

$$
\psi=R^{N} \varphi-i \sum_{j=1}^{N} R^{N-j+1} \varphi_{j} \tau-i(I-i A)^{-1}\left(I-R^{N}\right) p
$$

By Lemma 1, we get

$$
\begin{aligned}
p=T_{\tau}( & (I-i A) \psi-(I-i A) R^{N} \varphi \\
& \left.-\sum_{j=1}^{N}(I-i A) R^{N-j+1} \varphi_{j} \tau\right) .
\end{aligned}
$$

Using (9) and (14), we get

$$
\begin{aligned}
& u_{k}= R^{k} \varphi-i \sum_{j=1}^{k} R^{k-j+1} \varphi_{j} \tau \\
&+\sum_{j=1}^{k} R^{k-j+1} \tau T_{\tau}\left((I-i A) \psi-(I-i A) R^{N} \varphi\right. \\
& \\
&\left.\quad-\sum_{j=1}^{N}(I-i A) R^{N-j+1} \varphi_{j} \tau\right), \\
& 1 \leq k \leq N .
\end{aligned}
$$

Since

$$
\begin{aligned}
\sum_{j=1}^{k} R^{k-j+1} \tau & =(I-i A)^{-1}(I-R) \sum_{j=1}^{k} R^{k-j} \\
& =(I-i A)^{-1}\left(I-R^{k}\right)
\end{aligned}
$$

we have that

$$
\begin{aligned}
u_{k}= & R^{k} \varphi+\sum_{j=1}^{k} R^{k-j+1} \varphi_{j} \tau \\
& +\left(I-R^{k}\right) T_{\tau}\left(\psi-R^{N} \varphi-\sum_{j=1}^{N} R^{N-j+1} \varphi_{j} \tau\right), \\
& 1 \leq k \leq N .
\end{aligned}
$$

Hence, difference scheme (2) is uniquely solvable and for the solution, formulas (14) and (17) hold. 
Theorem 2. Suppose that the assumption of Lemma 1 holds. Let $\varphi, \psi \in D(A)$. Then, for the solution $\left(\left\{u_{k}\right\}_{k=1}^{N}, p\right)$ of difference scheme (2) in $C_{\tau}(H) \times H$, the estimates

$$
\begin{aligned}
\|p\|_{H} \leq M & {\left[\|A \varphi\|_{H}+\|A \psi\|_{H}\right.} \\
& \left.+\left\|\varphi_{1}\right\|_{H}+\max _{2 \leq k \leq N}\left\|\frac{\varphi_{k}-\varphi_{k-1}}{\tau}\right\|_{H}\right], \\
\left\|\left\{u_{k}\right\}_{k=1}^{N}\right\|_{C_{\tau}(H)} \leq & M\left[\|\varphi\|_{H}+\|\psi\|_{H}+\left\|\left\{\varphi_{k}\right\}_{k=1}^{N}\right\|_{C_{\tau}(H)}\right]
\end{aligned}
$$

hold, where $M$ is independent of $\tau, \varphi, \psi$, and $\left\{\varphi_{k}\right\}_{k=1}^{N}$.

Proof. From formulas (9) and (14), it follows that

$$
\begin{aligned}
p=T_{\tau} & {\left[A \psi-R^{N} A \varphi-\varphi_{N}+R^{N} \varphi_{1}\right.} \\
& \left.-\sum_{j=2}^{N} R^{N-j+1}\left(\varphi_{j-1}-\varphi_{j}\right)\right] .
\end{aligned}
$$

Using this formula, the triangle inequality, and estimate (6), we obtain

$$
\begin{aligned}
&\|p\|_{H} \leq\left\|T_{\tau}\right\|_{H \rightarrow H}\left(\|A \psi\|_{H}+\left\|R^{N}\right\|_{H \rightarrow H}\|A \varphi\|_{H}\right. \\
&+\sum_{j=2}^{N}\left\|R^{N-j+1}\right\|_{H \rightarrow H}\left\|\varphi_{j}-\varphi_{j-1}\right\|_{H} \\
&\left.+\left\|\varphi_{N}\right\|+\left\|R^{N}\right\|_{H \rightarrow H}\left\|\varphi_{1}\right\|_{H}\right) \\
& \leq M\left[\|A \varphi\|_{H}+\|A \psi\|_{H}+\left\|\left\{\varphi_{k}\right\}_{k=1}^{N}\right\|_{C_{\tau}^{(1)}(H)}\right] .
\end{aligned}
$$

Estimate (18) is proved. Using formula (17), the triangle inequality, and estimate (6), we obtain

$$
\begin{aligned}
\left\|u_{k}\right\|_{H} \leq & {\left[\left\|R^{k}\right\|_{H \rightarrow H}\|\varphi\|_{H}+\sum_{j=1}^{k}\left\|R^{k-j+1}\right\|_{H \rightarrow H}\left\|\varphi_{j}\right\|_{H} \tau\right.} \\
& +\left(1+\left\|R^{k}\right\|_{H \rightarrow H}\right)\left\|T_{\tau}\right\|_{H \rightarrow H} \\
& \times\left(\|\psi\|_{H}+\left\|R^{N}\right\|_{H \rightarrow H}\|\varphi\|_{H}\right. \\
& \left.\left.+\sum_{j=1}^{N}\left\|R^{N-j+1}\right\|_{H \rightarrow H}\left\|\varphi_{j}\right\|_{H} \tau\right)\right] \\
\leq & M\left[\|\varphi\|_{H}+\|\psi\|_{H}+\left\|\left\{\varphi_{k}\right\}_{k=1}^{N}\right\|_{C_{\tau}(H)}\right]
\end{aligned}
$$

for any $k$. From that, it follows estimate (19). This completes the proof of Theorem 2 .

\section{Applications}

Now, we consider the simple applications of main Theorem 2.

First, the boundary value problem for the Schrödinger equation

$$
\begin{gathered}
i u_{t}-\left(a(x) u_{x}\right)_{x}+\delta u+i u=p(x)+f(t, x), \\
0<t<T, \quad 0<x<1, \\
u(0, x)=\varphi(x), \quad u(T, x)=\psi(x), \quad 0 \leq x \leq 1, \\
u(t, 0)=u(t, 1), \quad u_{x}(t, 0)=u_{x}(t, 1), \quad 0 \leq t \leq T
\end{gathered}
$$

is considered. Problem (23) has a unique smooth solution $(u(t, x), p(x))$ for the smooth functions $a(x) \geq a>0, x \in$ $(0,1), \delta>0, a(1)=a(0), \varphi(x), \psi(x)(x \in[0,1])$, and $f(t, x)$ $(t \in(0, T), x \in(0,1))$. This allows us to reduce the boundary value problem (23) to the boundary value problem (1) in a Hilbert space $H=L_{2}[0,1]$ with a self-adjoint positive definite operator $A^{x}$ defined by formula

$$
A^{x} u(x)=-\left(a(x) u_{x}\right)_{x}+\delta u
$$

with domain

$$
\begin{gathered}
D\left(A^{x}\right)=\left\{u(x): u(x), u_{x}(x),\left(a(x) u_{x}\right)_{x} \in L_{2}[0,1],\right. \\
\left.u(1)=u(0), u_{x}(1)=u_{x}(0)\right\} .
\end{gathered}
$$

The discretization of problem (23) is carried out in two steps. In the first step, we define the grid space

$$
[0,1]_{h}=\left\{x=x_{n}: x_{n}=n h, 0 \leq n \leq M, M h=1\right\} .
$$

Let us introduce the Hilbert space $L_{2 h}=L_{2}\left([0,1]_{h}\right)$ of the grid functions

$$
\varphi^{h}(x)=\left\{\varphi_{n}\right\}_{1}^{M-1}
$$

defined on $[0,1]_{h}$, equipped with the norm

$$
\left\|\varphi^{h}\right\|_{L_{2 h}}=\left(\sum_{x \in[0,1]_{h}}|\varphi(x)|^{2} h\right)^{1 / 2} .
$$

To the differential operator $A^{x}$ defined by formula (24), we assign the difference operator $A_{h}^{x}$ by the formula

$$
A_{h}^{x} \varphi^{h}(x)=\left\{-\left(a(x) \varphi_{\bar{x}}\right)_{x, n}+\delta \varphi_{n}\right\}_{1}^{M-1}
$$

acting in the space of grid functions $\varphi^{h}(x)=\left\{\varphi_{n}\right\}_{1}^{M-1}$ satisfying the conditions $\varphi_{0}=\varphi_{M}, \varphi_{1}-\varphi_{0}=\varphi_{M}-\varphi_{M-1}$. It is well known that $A_{h}^{x}$ is a self-adjoint positive definite operator in $L_{2 h}$. With the help of $A_{h}^{x}$, we reach the boundary value problem

$$
\begin{array}{r}
i \frac{d u^{h}(t, x)}{d t}+A_{h}^{x} u^{h}(t, x)+i u^{h}(t, x)=p^{h}(x)+f^{h}(t, x), \\
0<t<T, \quad x \in[0,1]_{h}, \\
u^{h}(0, x)=\varphi^{h}(x), \quad u^{h}(T, x)=\psi^{h}(x), \quad x \in[0,1]_{h} .
\end{array}
$$


In the second step, we replace (30) with the difference scheme (2)

$$
\begin{gathered}
i \frac{u_{k}^{h}(x)-u_{k-1}^{h}(x)}{\tau}+A_{h}^{x} u_{k}^{h}(x)+i u_{k}^{h}(x)=p^{h}(x)+f_{k}^{h}(x), \\
f_{k}^{h}(x)=f^{h}\left(t_{k}, x\right), \quad t_{k}=k \tau, \quad N \tau=T, \\
1 \leq k \leq N, \quad x \in[0,1]_{h}, \\
u^{h}(0, x)=\varphi^{h}(x), \quad u^{h}(T, x)=\psi^{h}(x), \quad x \in[0,1]_{h} .
\end{gathered}
$$

Theorem 3. The solution pairs $\left(\left\{u_{k}^{h}(x)\right\}_{0}^{N}, p^{h}(x)\right)$ of problem (31) satisfy the stability estimates

$$
\begin{gathered}
\left\|p^{h}\right\|_{L_{2 h} \leq} \leq M_{1}\left[\left\|\varphi^{h}\right\|_{L_{2 h}}+\left\|A_{h}^{x} \varphi^{h}\right\|_{L_{2 h}}+\left\|\psi^{h}\right\|_{L_{2 h}}\right. \\
\left.+\left\|A_{h}^{x} \psi^{h}\right\|_{L_{2 h}}+\left\|\left\{f_{k}^{h}\right\}_{1}^{N}\right\|_{C_{\tau}^{(1)}\left(L_{2 h}\right)}\right], \\
\left\|\left\{u_{k}^{h}\right\}_{1}^{N}\right\|_{C_{\tau}\left(L_{2 h}\right)} \leq M_{2}\left[\left\|\varphi^{h}\right\|_{L_{2 h}}+\left\|\psi^{h}\right\|_{L_{2 h}}+\left\|\left\{f_{k}^{h}\right\}_{1}^{N}\right\|_{C_{\tau}\left(L_{2 h}\right)}\right],
\end{gathered}
$$

where $M_{1}$ and $M_{2}$ do not depend on $\varphi^{h}, \psi^{h}$, and $f_{k}^{h}, 1 \leq k \leq N$. Here, $C_{\tau}^{(1)}\left(L_{2 h}\right)$ is the grid space of grid functions $\left\{f_{k}^{h}\right\}_{1}^{N}$ defined on $[0, T]_{\tau} \times[0,1]_{h}$ with norm

$$
\begin{gathered}
\left\|\left\{f_{k}^{h}\right\}_{1}^{N}\right\|_{C_{\tau}^{(1)}\left(L_{2 h}\right)}=\left\|\left\{f_{k}^{h}\right\}_{1}^{N}\right\|_{C_{\tau}\left(L_{2 h}\right)}+\sup _{1 \leq k<k+r \leq N} \frac{\left\|f_{k+r}^{h}-f_{k}^{h}\right\|_{L_{2 h}}}{r \tau}, \\
\left\|\left\{f_{k}^{h}\right\}_{1}^{N}\right\|_{C_{\tau}\left(L_{2 h}\right)}=\max _{1 \leq k \leq N}\left\|f_{k}^{h}\right\|_{L_{2 h}} .
\end{gathered}
$$

The proof of Theorem 3 is based on formulas for $p^{h}(x)$ and $\left\{u_{k}^{h}(x)\right\}_{1}^{N}$ and the symmetry property of operator $A_{h}^{x}$.

Second, let $\Omega=\left(x=\left(x_{1}, \ldots, x_{n}\right): 0<x_{k}<1, k=1, \ldots\right.$, $n)$ be the unit open cube in the $n$-dimensional Euclidean space $\mathbb{R}^{n}$ with boundary $S, \bar{\Omega}=\Omega \cup S$. In $[0, T] \times \Omega$, the boundary value problem for the multidimensional Schrödinger equation

$$
\begin{gathered}
i \frac{\partial u(t, x)}{\partial t}-\sum_{r=1}^{n}\left(a_{r}(x) u_{x_{r}}\right)_{x_{r}}+i u=p(x)+f(t, x), \\
x=\left(x_{1}, \ldots, x_{n}\right) \in \Omega, \quad 0<t<T, \\
u(0, x)=\varphi(x), \quad u(T, x)=\psi(x), \quad x \in \bar{\Omega}, \\
u(t, x)=0, \quad x \in S, 0 \leq t \leq T
\end{gathered}
$$

is considered. Here, $a_{r}(x) \geq a>0(x \in \Omega), f(t, x)(t \in(0, T)$, $x \in \Omega)$, and $\varphi(x), \psi(x)(x \in \bar{\Omega})$ are given smooth functions.

We consider the Hilbert space $L_{2}(\bar{\Omega})$ of all square integrable functions defined on $\bar{\Omega}$, equipped with the norm

$$
\|f\|_{L_{2}(\bar{\Omega})}=\left(\int \cdots \int_{x \in \bar{\Omega}}|f(x)|^{2} d x_{1} \cdots d x_{n}\right)^{1 / 2} .
$$

Problem (34) has a unique smooth solution $(u(t, x), p(x))$ for the smooth functions $\varphi(x), \psi(x), a_{r}(x)$, and $f(t, x)$. This allows us to reduce the problem (34) to the boundary value problem (1) in the Hilbert space $H=L_{2}(\bar{\Omega})$ with a selfadjoint positive definite operator $A^{x}$ defined by the formula

$$
A^{x} u(x)=-\sum_{r=1}^{n}\left(a_{r}(x) u_{x_{r}}\right)_{x_{r}}
$$

with domain

$$
\begin{gathered}
D\left(A^{x}\right)=\left\{u(x): u(x), u_{x_{r}}(x),\left(a_{r}(x) u_{x_{r}}\right)_{x_{r}} \in L_{2}(\bar{\Omega}),\right. \\
1 \leq r \leq n, u(x)=0, x \in S\} .
\end{gathered}
$$

The discretization of problem (34) is carried out in two steps. In the first step, we define the grid space

$$
\begin{gathered}
\bar{\Omega}_{h}=\left\{x=x_{r}=\left(h_{1} j_{1}, \ldots, h_{h} j_{n}\right),\right. \\
j=\left(j_{1}, \ldots, j_{h}\right), 0 \leq j_{r} \leq N_{r}, \\
\left.N_{r} h_{r}=1, r=1, \ldots, n\right\}, \\
\Omega_{h}=\bar{\Omega}_{h} \cap \Omega, \quad S_{h}=\bar{\Omega}_{h} \cap S
\end{gathered}
$$

and introduce the Hilbert space $L_{2 h}=L_{2}\left(\bar{\Omega}_{h}\right)$ of the grid functions

$$
\varphi^{h}(x)=\left\{\varphi\left(h_{1} j_{1}, \ldots, h_{n} j_{n}\right)\right\}
$$

defined on $\bar{\Omega}_{h}$, equipped with the norm

$$
\left\|\varphi^{h}\right\|_{L_{2 h}}=\left(\sum_{x \in \Omega_{h}}\left|\varphi^{h}(x)\right|^{2} h_{1} \cdots h_{n}\right)^{1 / 2} .
$$

To the differential operator $A^{x}$ defined by formula (36), we assign the difference operator $A_{h}^{x}$ by the formula

$$
A_{h}^{x} u^{h}=-\sum_{r=1}^{n}\left(\alpha_{r}(x) u_{x_{r}}^{h}\right)_{x_{r}, j_{r}},
$$

where $A_{h}^{x}$ is known as self-adjoint positive definite operator in $L_{2 h}$, acting in the space of grid functions $u^{h}(x)$ satisfying the conditions $u^{h}(x)=0$ for all $x \in S_{h}$. With the help of the difference operator $A_{h}^{x}$, we arrive to the following boundary value problem:

$$
\begin{array}{r}
i u_{t}^{h}(t, x)+A_{h}^{x} u^{h}(t, x)+i u^{h}(t, x)=p^{h}(x)+f^{h}(t, x), \\
0<t<T, \quad x \in \Omega_{h}, \\
u^{h}(0, x)=\varphi^{h}(x), \quad u^{h}(T, x)=\psi^{h}(x), \quad x \in \Omega_{h}
\end{array}
$$

for an infinite system of ordinary differential equations. 
The first order of accuracy difference scheme for the solution of problem (42) is

$$
\begin{gathered}
i \frac{u_{k}^{h}(x)-u_{k-1}^{h}(x)}{\tau}+A_{h}^{x} u_{k}^{h}(x)+i u_{k}^{h}(x) \\
=p^{h}(x)+f_{k}^{h}(x), \\
f_{k}^{h}(x)=f^{h}\left(t_{k}, x\right), \quad t_{k}=k \tau, \quad N \tau=T, \\
1 \leq k \leq N, \quad x \in \Omega_{h}, \\
u^{h}(0, x)=\varphi^{h}(x), \quad u^{h}(T, x)=\psi^{h}(x), \quad x \in \Omega_{h} .
\end{gathered}
$$

Theorem 4. The solution pairs $\left(\left\{u_{k}^{h}(x)\right\}_{0}^{N}, p^{h}(x)\right)$ of problem (43) satisfy the stability estimates

$$
\begin{gathered}
\left\|p^{h}\right\|_{L_{2 h}} \leq M_{1}\left[\left\|\varphi^{h}\right\|_{L_{2 h}}+\left\|A_{h}^{x} \varphi^{h}\right\|_{L_{2 h}}+\left\|\psi^{h}\right\|_{L_{2 h}}\right. \\
\left.+\left\|A_{h}^{x} \psi^{h}\right\|_{L_{2 h}}+\left\|\left\{f_{k}^{h}\right\}_{1}^{N}\right\|_{C_{\tau}^{(1)}\left(L_{2 h}\right)}\right], \\
\left\|\left\{u_{k}^{h}\right\}_{1}^{N}\right\|_{C_{\tau}\left(L_{2 h}\right)} \leq M_{2}\left[\left\|\varphi^{h}\right\|_{L_{2 h}}+\left\|\psi^{h}\right\|_{L_{2 h}}+\left\|\left\{f_{k}^{h}\right\}_{1}^{N}\right\|_{C_{\tau}\left(L_{2 h}\right)}\right],
\end{gathered}
$$

where $M_{1}$ and $M_{2}$ do not depend on $\varphi^{h}, \psi^{h}$, and $f_{k}^{h}, 1 \leq k \leq N$. Here, $C_{\tau}^{(1)}\left(L_{2 h}\right)$ is the grid space of grid functions $\left\{f_{k}^{h}\right\}_{1}^{N}$ defined on $[0, T]_{\tau} \times \overline{\Omega_{h}}$ with norm

$$
\begin{gathered}
\left\|\left\{f_{k}^{h}\right\}_{1}^{N}\right\|_{C_{\tau}^{(1)}\left(L_{2 h}\right)}=\left\|\left\{f_{k}^{h}\right\}_{1}^{N}\right\|_{C_{\tau}\left(L_{2 h}\right)}+\sup _{1 \leq k<k+r \leq N} \frac{\left\|f_{k+r}^{h}-f_{k}^{h}\right\|_{L_{2 h}}}{r \tau}, \\
\left\|\left\{f_{k}^{h}\right\}_{1}^{N}\right\|_{C_{\tau}\left(L_{2 h}\right)}=\max _{1 \leq k \leq N}\left\|f_{k}^{h}\right\|_{L_{2 h}} .
\end{gathered}
$$

The proof of Theorem 4 is based on Theorem 3 and the symmetry property of the operator $A_{h}^{x}$ is defined by formula (34) and the following theorem on the coercivity inequality for the solution of the elliptic difference problem in $L_{2 h}$.

Theorem 5. For the solutions of the elliptic difference problem [26]

$$
\begin{gathered}
A_{h}^{x} u^{h}(x)=\omega^{h}(x), \quad x \in \Omega_{h}, \\
u^{h}(x)=0, \quad x \in S_{h},
\end{gathered}
$$

the following coercivity inequality holds:

$$
\sum_{r=1}^{n}\left\|u_{x_{r} x_{\bar{r}}}^{h}\right\|_{L_{2 h}} \leq M\left\|\omega^{h}\right\|_{L_{2 h}}
$$

where $M$ does not depend on $h$ and $\omega^{h}$.
Third, in $[0, T] \times \Omega$, the boundary value problem for the multidimensional Schrödinger equation

$$
\begin{gathered}
i \frac{\partial u(t, x)}{\partial t}-\sum_{r=1}^{n}\left(a_{r}(x) u_{x_{r}}\right)_{x_{r}}+\delta u+i u=p(x)+f(t, x), \\
x=\left(x_{1}, \ldots, x_{n}\right) \in \Omega, \quad 0<t<T, \quad x \in \bar{\Omega}, \\
u(0, x)=\varphi(x), \quad u(T, x)=\psi(x), \quad x \in S, \quad 0 \leq t \leq T
\end{gathered}
$$

with the Neumann condition is considered. Here, $\vec{n}$ is the normal vector to $S, \delta>0$, and $a_{r}(x) \geq a>0(x \in \Omega), f(t, x)$ $(t \in(0, T), x \in \Omega)$, and $\varphi(x), \psi(x)(x \in \bar{\Omega})$ are given smooth functions.

Problem (48) has a unique smooth solution $(u(t, x), p(x))$ for the smooth functions $\varphi(x), \psi(x), a_{r}(x)$, and $f(t, x)$. This allows us to reduce the problem (48) to the boundary value problem (1) in the Hilbert space $H=L_{2}(\bar{\Omega})$ with a selfadjoint positive definite operator $A^{x}$ defined by formula

$$
A^{x} u(x)=-\sum_{r=1}^{n}\left(a_{r}(x) u_{x_{r}}\right)_{x_{r}}+\delta u
$$

with domain

$$
\begin{gathered}
D\left(A^{x}\right)=\left\{u(x): u(x), u_{x_{r}}(x),\left(a_{r}(x) u_{x_{r}}\right)_{x_{r}} \in L_{2}(\bar{\Omega}),\right. \\
\left.1 \leq r \leq n, \frac{\partial u(x)}{\partial \vec{n}}=0, x \in S\right\} .
\end{gathered}
$$

The discretization of problem (48) is carried out in two steps. In the first step, we define the difference operator $A_{h}^{x}$ by the formula

$$
A_{h}^{x} u^{h}=-\sum_{r=1}^{n}\left(\alpha_{r}(x) u_{x_{r}}^{h}\right)_{x_{r}, j_{r}}+\delta u^{h}
$$

where $A_{h}^{x}$ is known as self-adjoint positive definite operator in $L_{2 h}$, acting in the space of grid functions $u^{h}(x)$ satisfying the conditions $D^{h} u^{h}(x)=0$ for all $x \in S_{h}$. Here, $D^{h}$ is the approximation of the operator $\partial \cdot / \partial \vec{n}$. With the help of the difference operator $A_{h}^{x}$, we arrive to the following boundary value problem:

$$
\begin{array}{r}
i u_{t}^{h}(t, x)+A_{h}^{x} u^{h}(t, x)+i u^{h}(t, x)=p^{h}(x)+f^{h}(t, x), \\
0<t<T, \quad x \in \Omega_{h}, \\
u^{h}(0, x)=\varphi^{h}(x), \quad u^{h}(T, x)=\psi^{h}(x), \quad x \in \Omega_{h}
\end{array}
$$

for an infinite system of ordinary differential equations. 
The first order of accuracy difference scheme for the solution of problem (52) is

$$
\begin{gathered}
i \frac{u_{k}^{h}(x)-u_{k-1}^{h}(x)}{\tau}+A_{h}^{x} u_{k}^{h}(x)+i u_{k}^{h}(x)=p^{h}(x)+f_{k}^{h}(x), \\
f_{k}^{h}(x)=f^{h}\left(t_{k}, x\right), \quad t_{k}=k \tau, \quad N \tau=T, \\
1 \leq k \leq N, \quad x \in \Omega_{h}, \\
u^{h}(0, x)=\varphi^{h}(x), \quad u^{h}(T, x)=\psi^{h}(x), \quad x \in \Omega_{h} .
\end{gathered}
$$

Theorem 6. The solution pairs $\left(\left\{u_{k}^{h}(x)\right\}_{0}^{N}, p^{h}(x)\right)$ of problem (53) satisfy the stability estimates

$$
\begin{gathered}
\left\|p^{h}\right\|_{L_{2 h}} \leq M_{1}\left[\left\|\varphi^{h}\right\|_{L_{2 h}}+\left\|A_{h}^{x} \varphi^{h}\right\|_{L_{2 h}}+\left\|\psi^{h}\right\|_{L_{2 h}}\right. \\
\left.+\left\|A_{h}^{x} \psi^{h}\right\|_{L_{2 h}}+\left\|\left\{f_{k}^{h}\right\}_{1}^{N}\right\|_{C_{\tau}^{(1)}\left(L_{2 h}\right)}\right], \\
\left\|\left\{u_{k}^{h}\right\}_{1}^{N}\right\|_{C_{\tau}\left(L_{2 h}\right)} \leq M_{2}\left[\left\|\varphi^{h}\right\|_{L_{2 h}}+\left\|\psi^{h}\right\|_{L_{2 h}}+\left\|\left\{f_{k}^{h}\right\}_{1}^{N}\right\|_{C_{\tau}\left(L_{2 h}\right)}\right],
\end{gathered}
$$

where $M_{1}$ and $M_{2}$ do not depend on $\varphi^{h}, \psi^{h}$, and $f_{k}^{h}, 1 \leq k \leq N$.

The proof of Theorem 6 is based on Theorem 2 and the symmetry property of the operator $A_{h}^{x}$ is defined by formula (51) and the following theorem on the coercivity inequality for the solution of the elliptic difference problem in $L_{2 h}$.

Theorem 7. For the solution of the elliptic difference problem [26]

$$
\begin{gathered}
A_{h}^{x} u^{h}(x)=\omega^{h}(x), \quad x \in \Omega_{h}, \\
D^{h} u^{h}(x)=0, \quad x \in S_{h},
\end{gathered}
$$

the following coercivity inequality holds:

$$
\sum_{r=1}^{n}\left\|u_{x_{r} x_{\bar{r}}}^{h}\right\|_{L_{2 h}} \leq M\left\|\omega^{h}\right\|_{L_{2 h}},
$$

where $M$ does not depend on $h$ and $\omega^{h}$.

\section{Numerical Results}

In present section, for numerical analysis, the following boundary value problem

$$
\begin{array}{r}
i \frac{\partial u(t, x)}{\partial t}-\frac{\partial^{2} u(t, x)}{\partial x^{2}}+i u(t, x)=p(x)+f(t, x), \\
x \in(0, \pi), \quad t \in(0,1), \\
u(0, x)=\sin x, \quad u(1, x)=e^{-1} \sin x, \quad x \in[0, \pi], \\
u(t, 0)=u(t, \pi)=0, \quad t \in[0,1]
\end{array}
$$

is considered. The exact solution of problem $(57)$ is $u(t, x)=$ $e^{-t} \sin x$ and $p(x)=\sin x$.

The first order of accuracy difference scheme

$$
\begin{gathered}
i \frac{u_{n}^{k}-u_{n}^{k-1}}{\tau}-\frac{u_{n+1}^{k}-2 u_{n}^{k}+u_{n-1}^{k}}{h^{2}}+i u_{n}^{k}=\varphi_{n}^{k}+p\left(x_{n}\right), \\
1 \leq k \leq N, \quad 1 \leq n \leq M-1, \\
\varphi_{n}^{k}=f\left(t_{k}, x_{n}\right)=\left(e^{-t_{k}}-1\right) \sin x_{n}, \\
t_{k}=k \tau, \quad 0 \leq k \leq N, \quad N \tau=1, \\
x_{n}=n h, \quad 1 \leq n \leq M-1, \quad M h=\pi, \\
u_{n}^{0}=\sin \left(x_{n}\right), \quad u_{n}^{N}=e^{-1} \sin \left(x_{n}\right), \quad x_{n}=n h, 0 \leq n \leq M, \\
u_{0}^{k}=u_{M}^{k}=0, \quad 0 \leq k \leq N
\end{gathered}
$$

for the numerical solution of problem (57) is constructed.

For obtaining the values of $p\left(x_{n}\right)$ at the grid points, we will use the following equation:

$$
\begin{array}{r}
p\left(x_{n}\right)=-e^{-1} \frac{\sin \left(x_{n+1}\right)-2 \sin \left(x_{n}\right)+\sin \left(x_{n-1}\right)}{h^{2}} \\
+i e^{-1} \sin \left(x_{n}\right)+\frac{v_{n+1}^{N}-2 v_{n}^{N}+v_{n-1}^{N}}{h^{2}}-i v_{n}^{N}, \\
x_{n}=n h, \quad 1 \leq n \leq M-1,
\end{array}
$$

where $v_{s}^{k}, s=n \pm 1$, and $n$ is the solution of the first order of accuracy difference scheme

$$
\begin{gathered}
i \frac{v_{n}^{k}-v_{n}^{k-1}}{\tau}-\frac{v_{n+1}^{k}-2 v_{n}^{k}+v_{n-1}^{k}}{h^{2}}+i v_{n}^{k}=\varphi_{n}^{k}, \\
\varphi_{n}^{k}=f\left(t_{k}, x_{n}\right), \quad t_{k}=k \tau, 1 \leq k \leq N, N \tau=1, \\
x_{n}=n h, \quad 1 \leq n \leq M-1, \quad M h=\pi, \\
v_{n}^{N}-v_{n}^{0}=\left(e^{-1}-1\right) \sin \left(x_{n}\right), \quad x_{n}=n h, 0 \leq n \leq M, \\
v_{0}^{k}=v_{M}^{k}=0, \quad 0 \leq k \leq N
\end{gathered}
$$

generated by difference scheme (58).

Using the difference scheme $(60)$, we obtain $(N+1) \times$ $(M+1)$ system of linear equations and we can write them in the matrix form as

$$
\begin{gathered}
A v_{n+1}+B v_{n}+C v_{n-1}=R \varphi_{n}, \quad 1 \leq n \leq M-1, \\
v_{0}=v_{M}=\widetilde{0},
\end{gathered}
$$


where

$$
\begin{aligned}
C=A & =\left[\begin{array}{cccccccc}
0 & 0 & 0 & \cdot & 0 & 0 & 0 & 0 \\
0 & x & 0 & \cdot & 0 & 0 & 0 & 0 \\
0 & 0 & x & \cdot & 0 & 0 & 0 & 0 \\
\cdot & \cdot & \cdot & \cdot & \cdot & \cdot & \cdot & \cdot \\
0 & 0 & 0 & \cdot & 0 & 0 & x & 0 \\
0 & 0 & 0 & \cdot & 0 & 0 & 0 & x
\end{array}\right]_{(N+1) \times(N+1)} \\
B & =\left[\begin{array}{ccccccc}
-1 & 0 & 0 & \cdot & 0 & 0 & 1 \\
y & z & 0 & \cdot & 0 & 0 & 0 \\
0 & y & z & \cdot & 0 & 0 & 0 \\
\cdot & \cdot & \cdot & \cdot & \cdot & \cdot & \cdot \\
0 & 0 & 0 & \cdot & y & z & 0 \\
0 & 0 & 0 & \cdot & 0 & y & z
\end{array}\right]_{(N+1) \times(N+1)}
\end{aligned}
$$

Here,

$$
\begin{gathered}
x=-\frac{1}{h^{2}}, \quad y=-\frac{i}{\tau}, \quad z=\frac{i}{\tau}+\frac{2}{h^{2}}+i, \\
v_{s}=\left[\begin{array}{c}
v_{s}^{0} \\
\vdots \\
v_{s}^{N}
\end{array}\right]_{(N+1) \times 1} \quad \text { for } s=n+1, n, n-1, \\
\varphi_{n}=\left[\begin{array}{c}
\left(e^{-1}-1\right) \sin x_{n} \\
\varphi_{n}^{1} \\
\vdots \\
\varphi_{n}^{N-1} \\
\varphi_{n}^{N}
\end{array}\right]_{(N+1) \times 1} .
\end{gathered}
$$

So, we have the second-order difference equation with respect to $n$ with matrix coefficients. Using the modified Gauss elimination method, we can obtain $v_{n}^{k}, 0 \leq k \leq N, 0 \leq n \leq M$.

For the solution of the matrix equations, we seek the solution of the form

$$
\begin{gathered}
v_{n}=\alpha_{n+1} v_{n+1}+\beta_{n+1}, \quad n=M-1, \ldots, 2,1, \\
v_{M}=\widetilde{0},
\end{gathered}
$$

where $\alpha_{j}$ and $\beta_{j}, j=1, \ldots, M$, are calculated as

$$
\begin{gathered}
\alpha_{n+1}=-\left(B+C \alpha_{n}\right)^{-1}(A), \\
\beta_{n+1}=\left(B+C \alpha_{n}\right)^{-1}\left(D \varphi_{n}-C \beta_{n}\right),
\end{gathered}
$$

where $\alpha_{1}$ is $(N+1) \times(N+1)$ and $\beta_{1}$ is $(N+1) \times 1$ zero matrix.

Then, using (59), values of $p\left(x_{n}\right)$ at grid points are obtained. Replacing $p\left(x_{n}\right)$ in (58), we get $(N+1) \times(M+1)$ system of linear equations and it can be written in the matrix form

$$
\begin{gathered}
A_{2} u_{n+1}+B_{2} u_{n}+C_{2} u_{n-1}=R \theta_{n}, \quad 1 \leq n \leq M-1, \\
1 u_{0}=u_{M}=\widetilde{0},
\end{gathered}
$$

TABLE 1: Error analysis for the exact solution $u(t, x)$.

\begin{tabular}{lccc}
\hline Method & $N=M=20$ & $N=M=40$ & $N=M=80$ \\
\hline $\begin{array}{l}\text { 1st order of } \\
\text { accuracy d.s. }\end{array}$ & 0.0024 & 0.0012 & $6.0463 \times 10^{-4}$ \\
\hline
\end{tabular}

where

$$
\begin{gathered}
C_{2}=C, \quad \begin{array}{l}
A_{2}=A, \\
B_{2}=\left[\begin{array}{ccccccc}
1 & 0 & 0 & \cdot & 0 & 0 & 0 \\
y & z & 0 & \cdot & 0 & 0 & 0 \\
0 & y & z & \cdot & 0 & 0 & 0 \\
\cdot & \cdot & \cdot & \cdot & \cdot & \cdot & \cdot \\
0 & 0 & 0 & \cdot & y & z & 0 \\
0 & 0 & 0 & \cdot & 0 & y & z
\end{array}\right]_{(N+1) \times(N+1)}
\end{array} .
\end{gathered}
$$

Here,

$$
\begin{gathered}
y=-\frac{i}{\tau}, \quad z=\frac{i}{\tau}+\frac{2}{h^{2}}+i, \\
u_{s}=\left[\begin{array}{c}
u_{s}^{0} \\
\vdots \\
u_{s}^{N}
\end{array}\right]_{(N+1) \times 1} \quad \text { for } s=n+1, n, n-1, \\
\theta_{n}=\left[\begin{array}{c}
\sin x_{n} \\
\varphi_{n}^{1}+p\left(x_{n}\right) \\
\vdots \\
\varphi_{n}^{N-1}+p\left(x_{n}\right) \\
\varphi_{n}^{N}+p\left(x_{n}\right)
\end{array}\right]_{(N+1) \times 1} .
\end{gathered}
$$

Using the modified Gauss elimination method again, we can obtain $u_{n}^{k}, 0 \leq k \leq N, 0 \leq n \leq M$.

We will give the results of the numerical analysis. The numerical solutions are recorded for different values of $N$ and $M$ and $u_{n}^{k}$ represents the numerical solutions of the difference scheme at $\left(t_{k}, x_{n}\right)$. Table 1 is constructed for $N=M=20$, 40 , and 80 , respectively and the errors are computed by the following formula:

$$
E=\max _{1 \leq k \leq N}\left\{\sum_{n=1}^{M}\left|u\left(t_{k}, x_{n}\right)-u_{n}^{k}\right|^{2} h\right\}^{1 / 2} .
$$

For their comparison, Table 2 is constructed when errors are computed by

$$
E=\max _{\substack{1 \leq k \leq N \\ 1 \leq n \leq M}}\left|u\left(t_{k}, x_{n}\right)-u_{n}^{k}\right| .
$$

Table 3 is constructed for the error of $p(x)$ at the nodes in maximum norm.

\section{Conclusion}

In the present study, the well-posedness of difference problem for the approximate solution of determination of a control 
TABLE 2: Error analysis for the exact solution $u(t, x)$.

\begin{tabular}{lccc}
\hline Method & $N=M=20$ & $N=M=40$ & $N=M=80$ \\
\hline $\begin{array}{l}\text { 1st order of } \\
\text { accuracy d.s. }\end{array}$ & 0.0019 & $9.5692 \times 10^{-4}$ & $4.8241 \times 10^{-4}$ \\
\hline
\end{tabular}

TABLE 3: Error analysis for $p(x)$.

\begin{tabular}{lccc}
\hline Method & $N=20$ & $N=40$ & $N=80$ \\
\hline 1st order of accuracy d.s. & 0.0145 & 0.0072 & 0.0036 \\
\hline
\end{tabular}

parameter for the Schrödinger equation is established. In practice, the stability inequalities for the solution of difference schemes of the approximate solution of three different types of control parameter problems are obtained. The well-posedness of the boundary value problem (1) is established. The stability inequalities for the solution of difference schemes for three different types of control parameter problems for the Schrödinger equation are obtained. Moreover, applying the result of the monograph [15], the high order of accuracy single-step difference schemes for the numerical solution of the boundary value problem (1) can be presented. Of course, the stability inequalities for the solution of these difference schemes have been established without any assumptions about the grid steps.

\section{Conflict of Interests}

The authors declare that they have no conflict of interests.

\section{References}

[1] M. Dehghan, "Determination of a control parameter in the twodimensional diffusion equation," Applied Numerical Mathematics, vol. 37, no. 4, pp. 489-502, 2001.

[2] T. Kimura and T. Suzuki, "A parabolic inverse problem arising in a mathematical model for chromatography," SIAM Journal on Applied Mathematics, vol. 53, no. 6, pp. 1747-1761, 1993.

[3] Y. A. Gryazin, M. V. Klibanov, and T. R. Lucas, "Imaging the diffusion coefficient in a parabolic inverse problem in optical tomography," Inverse Problems, vol. 15, no. 2, pp. 373-397, 1999.

[4] Y. S. Eidelman, Boundary value problems for differential equations with parameters [Ph.D. thesis], Voronezh State University, Voronezh, Russia, 1984 (Russian).

[5] A. Hasanov, "Identification of unknown diffusion and convection coefficients in ion transport problems from flux data: an analytical approach," Journal of Mathematical Chemistry, vol. 48, no. 2, pp. 413-423, 2010.

[6] G. di Blasio and A. Lorenzi, "Identification problems for parabolic delay differential equations with measurement on the boundary," Journal of Inverse and Ill-Posed Problems, vol. 15, no. 7, pp. 709-734, 2007.

[7] D. Orlovsky and S. Piskarev, "On approximation of inverse problems for abstract elliptic problems," Journal of Inverse and Ill-Posed Problems, vol. 17, no. 8, pp. 765-782, 2009.

[8] D. Orlovsky and S. Piskarev, "The approximation of BitzadzeSamarsky type inverse problem for elliptic equations with Neumann conditions," Contemporary Analysis and Applied Mathematics, vol. 1, no. 2, pp. 118-131, 2013.
[9] A. Ashyralyev, "On the problem of determining the parameter of a parabolic equation," Ukrainian Mathematical Journal, vol. 62, no. 9, pp. 1397-1408, 2011.

[10] V. Serov and L. Päivärinta, "Inverse scattering problem for twodimensional Schrödinger operator," Journal of Inverse and IllPosed Problems, vol. 14, no. 3, pp. 295-305, 2006.

[11] A. Ashyralyev and B. Hicdurmaz, "A note on the fractional Schrödinger differential equations," Kybernetes, vol. 40, no. 5-6, pp. 736-750, 2011.

[12] A. Ashyralyev and B. Hicdurmaz, "On the numerical solution of fractional Schrödinger differential equations with the Dirichlet condition," International Journal of Computer Mathematics, vol. 89, no. 13-14, pp. 1927-1936, 2012.

[13] A. Ashyralyev, A. S. Erdogan, and O. Demirdag, "On the determination of the right-hand side in a parabolic equation," Applied Numerical Mathematics, vol. 62, no. 11, pp. 1672-1683, 2012.

[14] A. S. Erdogan and H. Uygun, "A note on the inverse problem for a fractional parabolic equation," Abstract and Applied Analysis, vol. 2012, Article ID 276080, 26 pages, 2012.

[15] Ch. Ashyralyyev and M. Dedeturk, "A finite difference method for the inverse elliptic problem with the Dirichlet condition," Contemporary Analysis and Applied Mathematics, vol. 1, no. 2, pp. 132-155, 2013.

[16] A. Ashyralyev and P. E. Sobolevskii, New Difference Schemes for Partial Differential Equations, vol. 148 of Operator Theory: Advances and Applications, Birkhäuser, Basel, Switzerland, 2004.

[17] S. Avdonin, S. Lenhart, and V. Protopopescu, "Solving the dynamical inverse problem for the Schrödinger equation by the boundary control method," Inverse Problems, vol. 18, no. 2, pp. 349-361, 2002.

[18] G. Eskin and J. Ralston, "Inverse scattering problem for the Schrödinger equation with magnetic potential at a fixed energy," Communications in Mathematical Physics, vol. 173, no. 1, pp. 199-224, 1995.

[19] A. Mercado, A. Osses, and L. Rosier, "Inverse problems for the Schrödinger equation via Carleman inequalities with degenerate weights," Inverse Problems, vol. 24, no. 1, Article ID 015017, 2008.

[20] T. Nadareishvili and A. Khelashvili, "Pragmatic SAE procedure in the Schrodinger equation for the inverse-square-like potentials," High Energy Physics - Theory, vol. 93, pp. 1-26, 2012.

[21] H. Nakatsuji, "Inverse Schrödinger equation and the exact wave function," Physical Review A, vol. 65, no. 5, pp. 1-15, 2002.

[22] A. Ashyralyev and A. Sirma, "Nonlocal boundary value problems for the Schrödinger equation," Computers \& Mathematics with Applications, vol. 55, no. 3, pp. 392-407, 2008.

[23] A. Ashyralyev and A. Sirma, "Modified Crank-Nicolson difference schemes for nonlocal boundary value problem for the Schrödinger equation," Discrete Dynamics in Nature and Society, vol. 2009, Article ID 584718, 15 pages, 2009.

[24] A. Ashyralyev and A. Sirma, "A note on the numerical solution of the semilinear Schrödinger equation," Nonlinear Analysis: Theory, Methods \& Applications, vol. 71, no. 12, pp. e2507-e2516, 2009.

[25] A. Ashyralyev and M. Urun, "Determination of a control parameter for the Schrödinger equation," Contemporary Analysis and Applied Mathematics, vol. 1, no. 2, pp. 156-166, 2013.

[26] P. E. Sobolevskii, Difference Methods for the Approximate Solution of Differential Equations, Izdatelstvo Voronezhskogo Gosud Universiteta, Voronezh, Russia, 1975 (Russian). 


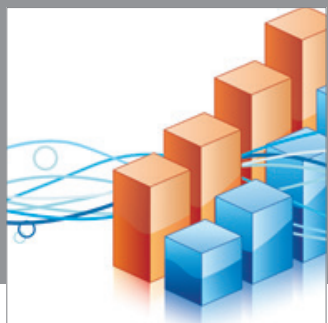

Advances in

Operations Research

mansans

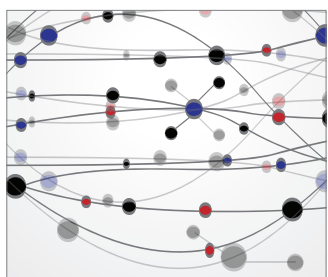

The Scientific World Journal
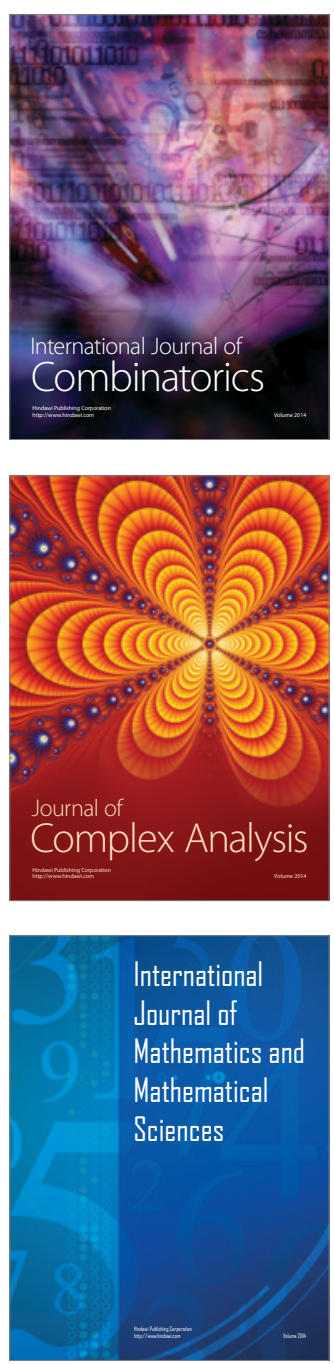
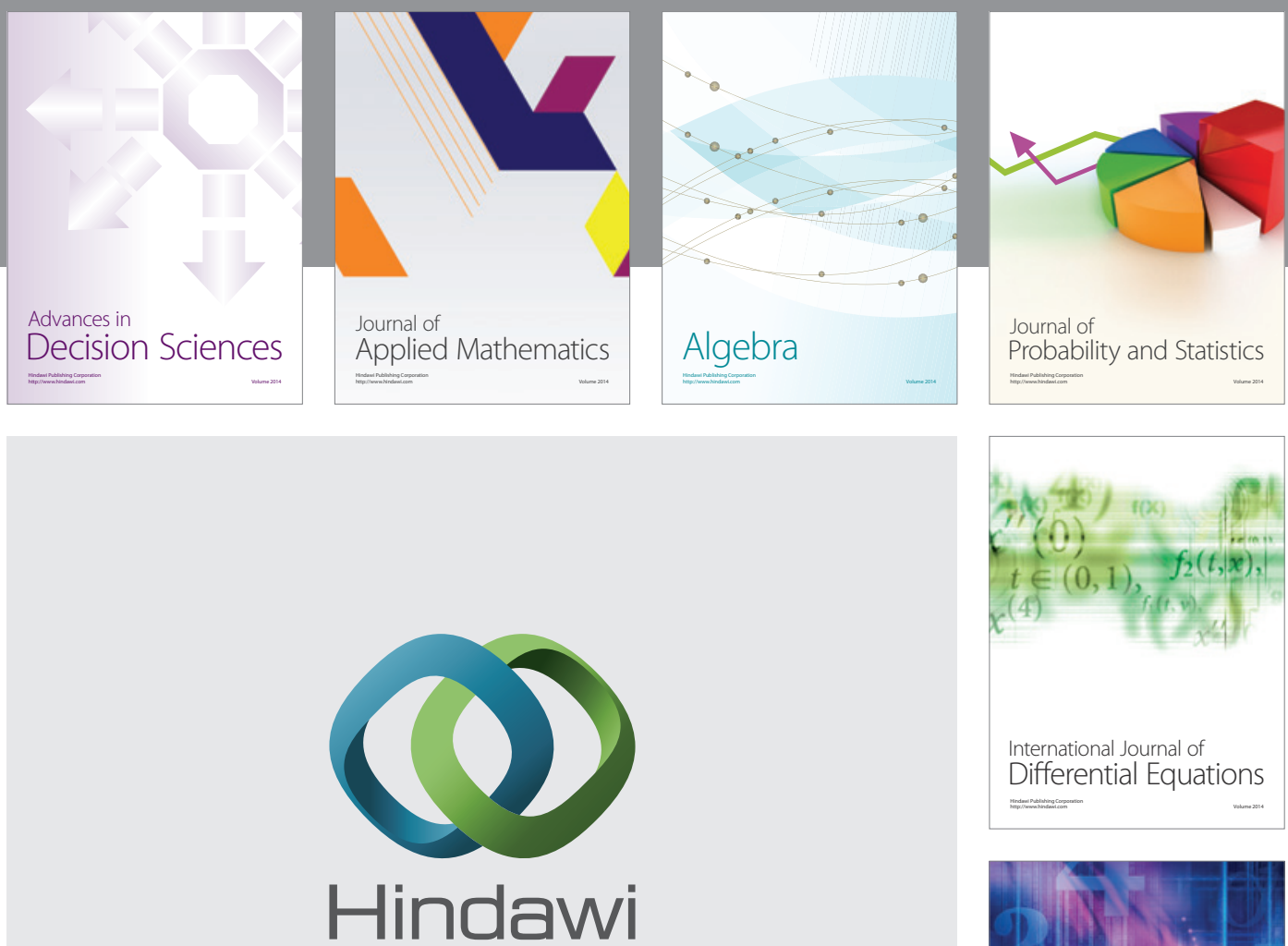

Submit your manuscripts at http://www.hindawi.com
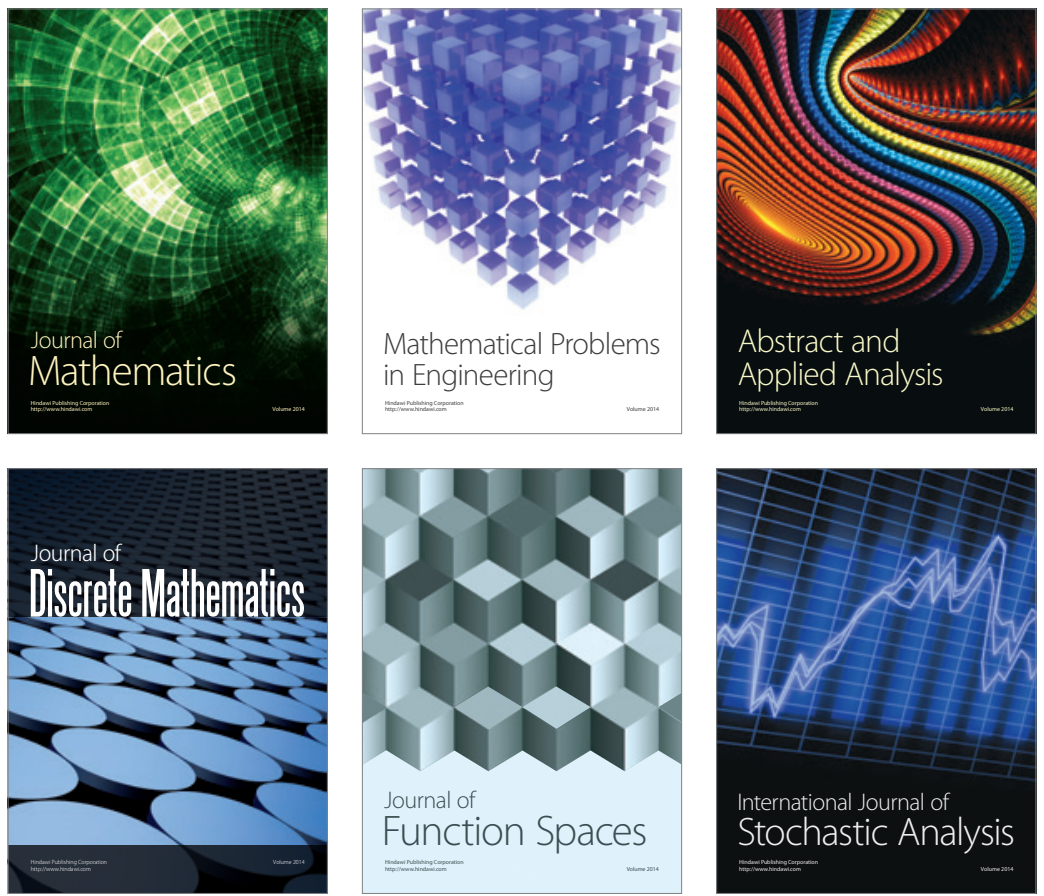

Journal of

Function Spaces

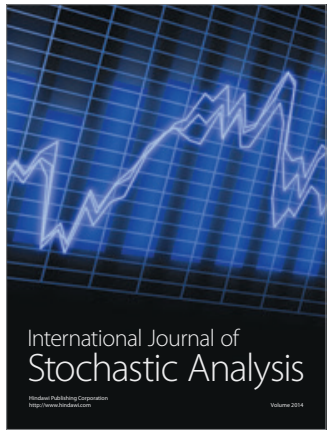

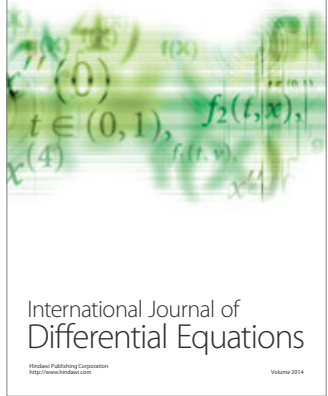
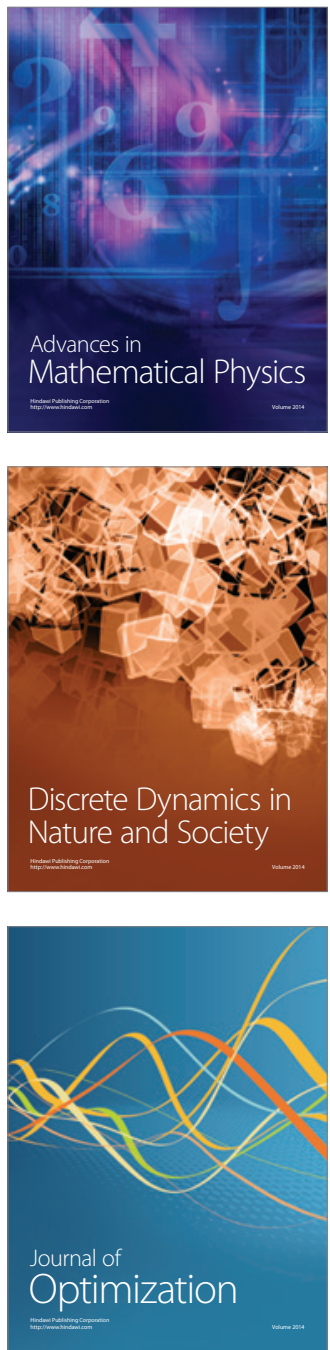